Bader B. Alsulays*, Md. Khalid Anwer, Mohammed F. Aldawsari, Alhussain Aodah, Elsadig Adam, Sultan Alshehri and Maged S. Abdel-Kader

\title{
Preparation and evaluation of a stable and sustained release of lansoprazole-loaded poly(D,L-lactide-co-glycolide) polymeric nanoparticles
}

https://doi.org/10.1515/polyeng-2019-0164

Received May 22, 2019; accepted July 21, 2019; previously published online September 10, 2019

Abstract: The aim of the study was to prepare lansoprazole (LNS)-loaded poly(D,L-lactide-co-glycolide) (PLGA) nanoparticles in order to improve the physicochemical stability associated with LNS. We synthesized LNS-loaded PLGA nanoparticles in the presence of magnesium oxide as alkalizer to improve the release of LNS and stability against photodegradation. The LNS-encapsulated PLGA nanoparticles were developed by the nanoprecipitation/ solvent evaporation method, resulting in a particle size of $246.7 \pm 3.4 \mathrm{~nm}$, polydispersity index (PDI) of 0.126 , percent drug entrapment (PDE) of $82.85 \pm 4.5 \%$, percent drug loading (PDL) of $3.54 \pm 0.34 \%$, and $\mathrm{pH}$ of $8.10 \pm 0.56$. The developed nanoparticles were further evaluated for in vitro release and resistance to photodegradation by NMR spectroscopy and LC-MS. The sustained release of the drug was confirmed after the encapsulation of LNS in the PLGA matrix. The protection of LNS in the PLGA matrix against photodegradation was confirmed by NMR and LC-MS studies. The LC-MS of UV-exposed samples of pure LNS and LNS-loaded PLGA nanoparticles at $254 \mathrm{~nm}$ showed the same $(\mathrm{M}+1)$ peak at $370 \mathrm{~m} / \mathrm{e}$, and the base peak at $369 \mathrm{~m} / \mathrm{e}$ accounted for the unchanged structure of LNS inside PLGA nanoparticles. Overall, it was proved

\footnotetext{
*Corresponding author: Bader B. Alsulays, Department of Pharmaceutics, College of Pharmacy, Prince Sattam Bin Abdulaziz University, Al-Kharj 11942, Saudi Arabia,

e-mail: b.alsulays@psau.edu.sa

Md. Khalid Anwer, Mohammed F. Aldawsari and Alhussain Aodah: Department of Pharmaceutics, College of Pharmacy, Prince Sattam Bin Abdulaziz University, Al-Kharj 11942, Saudi Arabia

Elsadig Adam: Department of Pharmaceutical Chemistry, College of Pharmacy, Prince Sattam Bin Abdulaziz University, Al-Kharj 11942, Saudi Arabia

Sultan Alshehri: Department of Pharmaceutics, College of Pharmacy, King Saud University, PO Box 2457, Riyadh, Saudi Arabia Maged S. Abdel-Kader: Department of Pharmacognosy, College of Pharmacy, Prince Sattam Bin Abdulaziz University, Al-Kharj 11942, Saudi Arabia; and Department of Pharmacognosy, College of Pharmacy, Alexandria University, Alexandria 21215, Egypt
}

that PLGA nanoparticles in the presence of magnesium oxide are an efficient carrier to deliver and protect LNS from physicochemical instability.

Keywords: lansoprazole; nanoparticles; PLGA; stability; sustained release.

\section{Introduction}

Lansoprazole (LNS), a substituted imidazole derivative with formula 3-\{[3-methyl4-(2,2,2-trifluoroethoxy)2-pyrid]-sulfinyl benzimidazole (Figure 1), belongs to the group of proton pump inhibitors (PPI), indicated for the treatment of gastric ulcer, duodenal ulcer, reflux esophagitis, and gastroesophageal reflux disease (GERD) [1-5]. LNS acts selectively on hydrogen/potassium ATPase, which inhibits the final enzymatic step of the acid secretory pathway of gastric parietal cells causing anti-secretory effect, raising the intragastric $\mathrm{pH}$ [6]. The major drawbacks for developing LNS formulations are related to their physicochemical instability to heat, light, and acidic media. Moreover, LNS has low aqueous solubility and shows marked inter-subject variation in absolute bioavailability [7-10]. The rate of LNS degradation under aqueous conditions increases with the decrease in $\mathrm{pH}$. The sulfoxide group in the LNS molecule is easily attacked by a proton, which reduces its medicinal usefulness [11, 12].

There is, therefore, a need to learn how to inhibit LNS degradation. Previously, we reported a method to prepare stable enteric-coated LNS tablets using an alkalizer [13]. Fang et al. [14] developed stable, Eudragit L/HPMCAS blend of enteric-coated LNS pellets. Lin and Duh [15] improved the stability of acid-labile LNS by developing a nanostructured lipid carrier for transdermal delivery. Long-term stable, spray-dried, LNS-loaded enteric microparticles were developed by Vora et al. [16] .

Poly(D,L-lactide-co-glycolide) (PLGA) is a pharmaceutically safe, FDA-approved polymer frequently used to enhance a drug's performance in terms of improvement of drug release, stability, bioavailability, and therapeutic efficacy of poorly water soluble drugs [17-19]. 


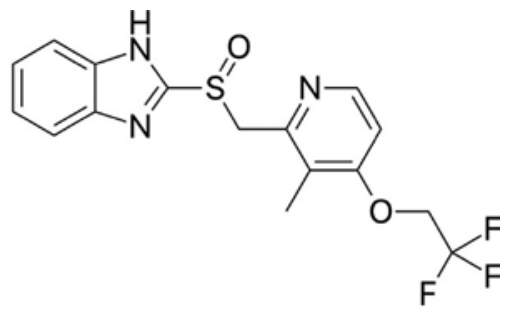

Figure 1: Chemical structure of LNS.

To extend the drug release time, reduce the frequency of administration, and improve the efficiency of the drug, as well as patient compliance, various drugs have been encapsulated in biodegradable PLGA nanoparticles (PLGA-NPs). The sustained release time of PLGA-NPs can be programmed by optimizing the particle size, morphology, and PLGA concentration. No previous studies have reported the role of PLGA-NPs on the photostabilization of LNS. The stabilization of the acid-labile drug LNS can be achieved using an alkalizer that provides a higher $\mathrm{pH}$. Magnesium oxide, magnesium carbonate, and sodium carbonate are the alkalizers used to protect the drug from an acidic environment $[13,20]$.

In this study, LNS-loaded PLGA polymeric nanoparticles were developed by nanoprecipitation/solvent evaporation. The alkalizer, magnesium oxide $(\mathrm{MgO})$, was also used in the preparation as a stabilizer and a dissolution enhancer for LNS. In addition, it modified the physicochemical properties of the drug [13]. Thus the goal of the current study was to develop stable LNS-loaded PLGA nanoparticles.

\section{Materials and methods}

\subsection{Materials}

LNS was purchased from Mesochem Technology (Beijing, China). PLGA (lactide/glycolide 50:50), Pluronic-127, polyvinyl alcohol (PVA), and magnesium oxide were obtained from Sigma-Aldrich (St. Louis, MO, USA). All solvents used for analysis were of HPLC grade. Milli Q-purified water was collected from the Department of Pharmacognosy, College of Pharmacy, Prince Sattam Bin Abdulaziz University, Al-Kharj, Saudi Arabia.

\subsection{Chromatographic analysis}

A slightly modified RP-HPLC method (a Waters HPLC system with a UV detector) was used to analyze the LNS samples during the experiments. The chromatographic column used was of $4.6 \mathrm{~mm} \times 250 \mathrm{~mm}$, C18, with $5 \mu \mathrm{m}$ particles. Briefly, the samples were analyzed at a flow rate of $1 \mathrm{ml} \mathrm{min}{ }^{-1}$ at room temperature under isocratic conditions. The mobile phase consisted of a mixture of triethyl amine buffer (pH 6.2) and acetonitrile (60:40, v/v). UV detection was done at $288 \mathrm{~nm}$ [21].

\subsection{Development of LNS-loaded PLGA nanoparticles}

LNS-loaded PLGA nanoparticles were prepared by the nanoprecipitation/solvent evaporation method [17]. Briefly, an accurately weighed amount of PLGA polymer (200 mg) was dissolved in ethyl acetate. LNS (20 mg) was dissolved in the polymeric solution, and the resulting organic phase was further added to an aqueous phase consisting Pluronic-127 (200 mg, as surfactant) and magnesium oxide (20 mg, as alkalizer) in 1\% w/v PVA slowly with a flow rate of $0.3 \mathrm{ml} \mathrm{min}{ }^{-1}$ at $25^{\circ} \mathrm{C}$. Ethyl acetate was evaporated at $40^{\circ} \mathrm{C}$ with continuous stirring with a magnetic stirrer for $24 \mathrm{~h}$. LNS-loaded PLGA nanoparticles were separated from the bulk aqueous phase by centrifugation at 12,000 rpm for $30 \mathrm{~min}$, followed by three times washing with cold distilled water and freeze-drying (Martin Christ Alpha-1-4LD freeze-drier, Osterode, Germany).

\subsection{Particle characterization}

Mean particle size, polydispersity index (PDI), and zeta potential (ZP) of the developed LNS-loaded PLGA nanoparticles were measured by dynamic light scattering (DLS). The developed nanoparticles were evaluated for size, PDI, and ZP by photon correlation spectroscopy using a Zetasizer Nano ZSP particle size analyzer (Malvern, UK). The LNS-loaded PLGA nanoparticle dispersion was diluted 100 times with distilled water and analyzed at room temperature $\left(25^{\circ} \mathrm{C}\right)$ with an angle of detection of $90^{\circ}$ [22].

\subsection{Measurement of percent drug entrapment (PDE) and percent drug loading (PDL)}

PDE and PDL of LNS in the nanoparticles were measured by centrifugation of the suspension of samples at $12,000 \mathrm{rpm}$ at $25^{\circ} \mathrm{C}$ for $20 \mathrm{~min}$ [22]. The amounts of unentrapped LNS were obtained in the supernatant and 
quantified by HPLC. PDE and PDL were calculated using the following equations:

$\mathrm{PDE}=[($ drug added - free drug $) /$ drug added $] \times 100$

$\mathrm{PDL}=$

[encapsulated drug / total nanoparticle weight] $\times 100$

\subsection{DSC thermal studies}

Differential scanning calorimetry (DSC) of the samples was carried out using a Shimadzu DSC-60 (Shimadzu Corporation, Tokyo, Japan) instrument to study their thermal behavior. The samples were weighed accurately $(5 \mathrm{mg})$ and placed in sealed aluminum crucibles. An empty sealed aluminum crucible/pan was used as the reference. The samples were analyzed at a scanning rate of $10^{\circ} \mathrm{C} \mathrm{min}{ }^{-1}$ from 25 to $250^{\circ} \mathrm{C}$ in an inert (nitrogen) environment (flow $\left.15 \mathrm{ml} \mathrm{min}^{-1}\right)$.

\subsection{Powder X-ray diffraction studies}

Powder X-ray diffraction (PXRD) patterns of free LNS and the LNS formulation were analyzed by X-ray diffractometer (Altima IV Regaco, Japan). The scanning rate was set at $4^{\circ} \mathrm{min}^{-1}$. The voltage/current used was $30 \mathrm{kV} / 25 \mathrm{~mA}$, and the target/filter (monochromator) was copper.

\subsection{Fourier transform infrared (FT-IR) spectroscopy}

The FT-IR spectra of LNS and the nanoparticles were recorded on an ALPHA FT-IR spectrometer (OPTIK, Billerica, MA, USA) using the $\mathrm{KBr}$ pellet technique. The samples were mixed with $\mathrm{KBr}$ (1:50) in a small, clean glass mortar, and then compressed to transparent pellets by applying a suitable pressure. The baseline was corrected by using a blank KBr pellet, and spectral scanning was done against a blank $\mathrm{KBr}$ pellet background in the wavenumber range $4000-400 \mathrm{~cm}^{-1}$.

\subsection{Scanning electron microscopy (SEM)}

The morphology of the prepared LNS-loaded PLGA nanoparticles was observed using a scanning electron microscope (Zeiss EVO LS10, Cambridge, UK). The suspended sample was vortexed for $1 \mathrm{~min}$, and one drop of the suspension was spread on a slide and dried. The sample was mounted on stubs using an adhesive carbon tape and coated under vacuum with gold in a sputter coater unit (Quorum Technologies Ltd, East Sussex, UK) in an argon atmosphere. The sample was then scanned and photomicrographs were taken.

\subsection{In vitro release studies}

The dissolution behavior of LNS-loaded PLGA NPs was studied in comparison with pure LNS. The in vitro release studies were carried out according to USP XXVI rotating method [23]. The powder samples, equivalent to $15 \mathrm{mg}$ of the pure LNS drug, were enclosed in soft gelatin capsules and placed in a basket containing $900 \mathrm{ml}$ of a phosphate buffer medium (pH 6.8 with $5 \mathrm{~mm}$ SLS) in order to maintain the sink condition. The stirring speed was $75 \mathrm{rpm}$, and the temperature was maintained at $37^{\circ} \mathrm{C}$. The samples $(5 \mathrm{ml})$ drawn at predetermined time intervals were analyzed by HPLC at $288 \mathrm{~nm}$ [21], and the same volume of fresh media was compensated. The data collected from in vitro release study was plotted and fitted to various kinetic models to know the release pattern of the drug from the PLGA matrix.

\subsection{Photodegradation studies}

Photodegradation studies under irradiation with UV light were performed using a UV lamp (São Paulo, Brazil). Stability of LNS and the nanoparticles was evaluated by exposing $500 \mathrm{mg}$ of powder in Petri-dish to short (254 nm) and long (366 nm) wavelength UV light. A $20 \mathrm{~W}$ short (254 nm) and long (366 nm) wavelength UV lamp was used for this study $[8,24,25]$. The samples were kept in the Petri-dish at a distance of $15 \mathrm{~cm}$ from the UV lamp for $72 \mathrm{~h}$.

\subsection{Photodegradation analysis by NMR spectroscopy and LC-MS}

${ }^{1} \mathrm{H}$ and ${ }^{13} \mathrm{C}$ NMR and 2D NMR experiments (COSY, HSQC, and $\mathrm{HMBC}$ ) were conducted using the standard Bruker program on an UltraShield Plus $500 \mathrm{MHz}$ (Bruker) spectrometer operating at $500 \mathrm{MHz}$ for protons and $125 \mathrm{MHz}$ for carbon atoms. The chemical shift values are reported in $\delta$ (ppm) relative to the residual solvent peak, and the coupling constants $(J)$ are reported in hertz (Hz). LC-MS analysis was performed on a Shimadzu LC-MS system 8030 operating in the electrospray ionization (ESI) positive 
mode. The drug sample was dissolved in a deionized water/methanol mixture (1:1) [26].

\section{Results and discussion}

\subsection{Particle size characterization, PDE, and PDL}

The mean particle size and PDI of the prepared formulations were determined as $246.7 \pm 3.4 \mathrm{~nm}$ and 0.126 , respectively. The PDE and PDL of LNS-loaded PLGA nanoparticles were measured as $82.85 \pm 4.5 \%$ and $3.45 \pm 0.34 \%$, respectively. The used PVA concentration $(1 \% \mathrm{w} / \mathrm{v})$ in the formulation might achieve complete surface coverage, resulting in optimal nanoparticle stabilization. A very low value of PDI indicates a narrow dispersion of particles, which means a uniform particle size distribution. The PDE and PDL were significantly high, revealing the maximum amount of drug encapsulation. The stable developed formulation was also confirmed by its $\mathrm{pH}$ value (measured $\mathrm{pH} 8.10 \pm 0.56$ ), as it is known that a basic condition favors stability.

\subsection{DSC studies}

The thermal behavior of LNS was compared with that of LNS-NPs using thermogravimetry in the temperature range $25-250^{\circ} \mathrm{C}$, as shown in Figure 2. The thermogram of the pure LNS showed a sharp endothermic peak corresponding to $180.55^{\circ} \mathrm{C}$, followed by rapid, sharp decomposition at $188^{\circ} \mathrm{C}$, which were in agreement with the values reported in the literature [8]. As we can see, in the DSC thermogram of the LNS formulation, the peak of LNS was completely absent. It may be due to the presence of PLGA polymer, Pluronic-127, and $\mathrm{MgO}$ in the formulation. This clearly showed that LNS was completely entrapped and protected inside the polymeric matrix.

\subsection{PXRD studies}

Figure 3 shows the PXRD patterns of pure LNS and the formulations. Pure LNS exhibited sharp peaks at $5.27^{\circ}, 14.25^{\circ}$, $16.88^{\circ}, 17.51^{\circ}, 22.31^{\circ}, 24.95^{\circ}$, and $25.91^{\circ}$. The LNS formulation exhibited broad and diffuse diffraction peaks, revealing its amorphous character after entrapment inside the polymer. This can be explained by the fact that the crystal structures of LNS, PLGA, Pluronic acid F-127, and magnesium oxide were destroyed during the formation of the nanoparticles, resulting in a reduction in the lattice density [16].

\subsection{FT-IR studies}

The characteristic FT-IR peaks of LNS were observed at $3227,2951,1616,1227$, and $1086 \mathrm{~cm}^{-1}$, which are due to bond

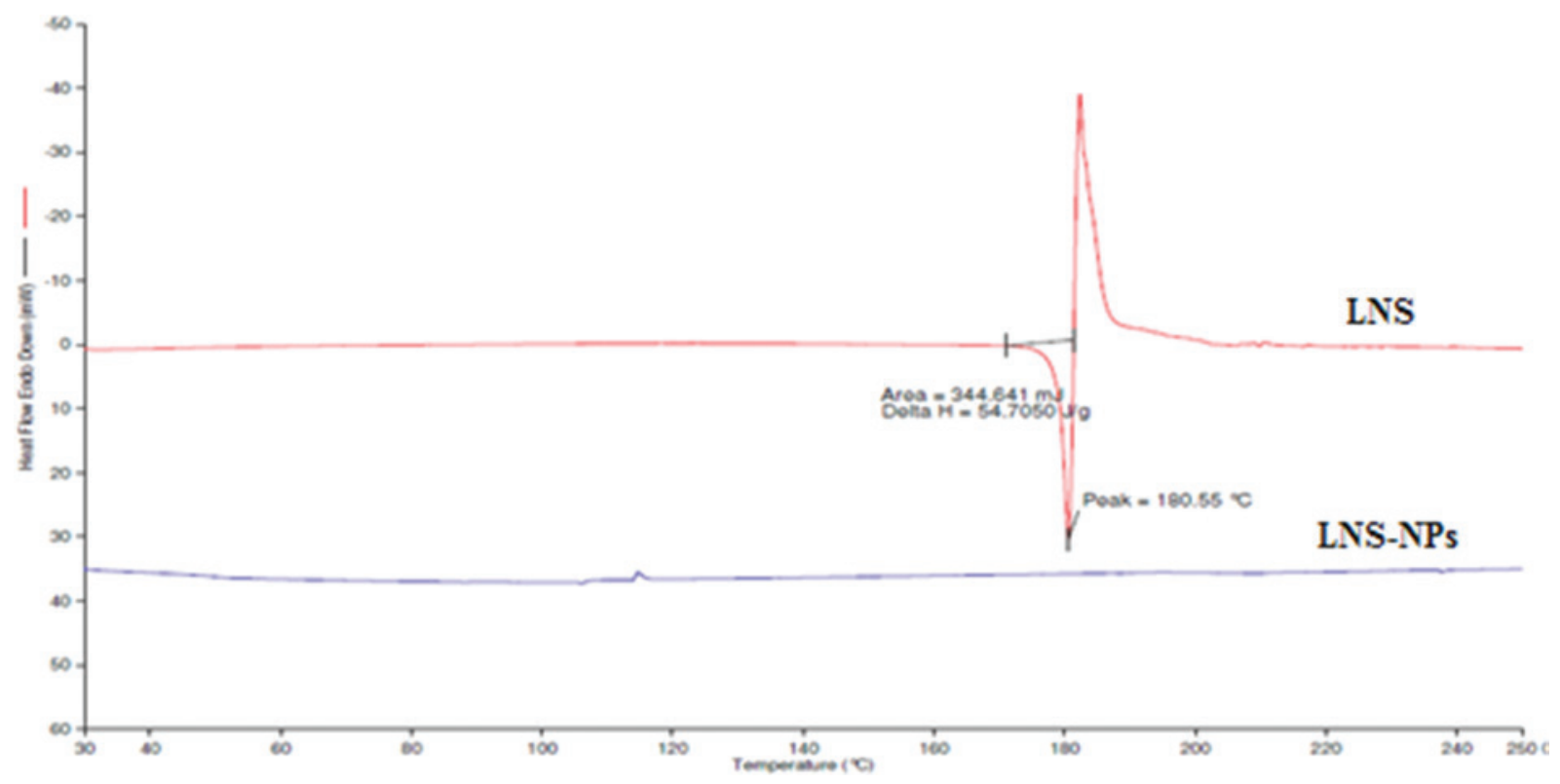

Figure 2: DSC thermogram of LNS and LNS-NPs. 

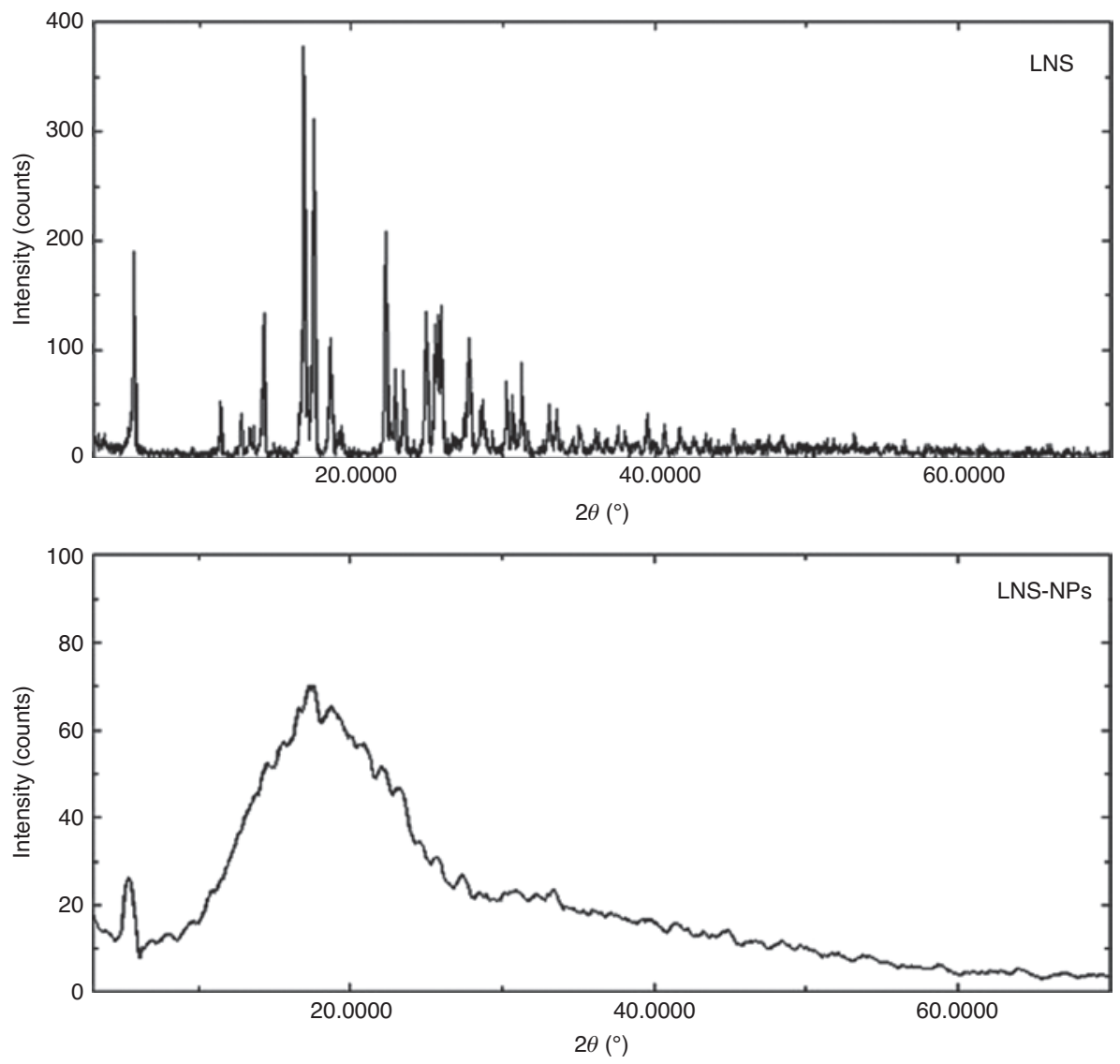

Figure 3: Powder XRD diffraction pattern of LNS and LNS-NPs.

stretching of $\mathrm{N}-\mathrm{H},-\mathrm{CH}_{2}, \mathrm{C}=\mathrm{N}, \mathrm{C}-\mathrm{N}$, ether bond, and $\mathrm{S}=\mathrm{O}$, respectively (Figure 4) [13]. Significant changes could be seen in the spectra of LNS-loaded PLGA nanoparticles, as all the characteristic peaks of LNS were reduced in intensity significantly in the fingerprint region, indicating the complete entrapment of LNS inside PLGA. This change also indicates hydrogen-bond formation between the drug and the polymer.

\subsection{SEM studies}

The SEM image of the LNS-loaded PLGA nanoparticles is shown in Figure 5. It can be seen from the image that the prepared nanoparticles are spherical with a smooth surface. From the image, it is clear that the nanoparticles are agglomerated, which might be due to the presence of the alkalizer (magnesium oxide) in the formulation. The result obtained through SEM showed an average particle size of $205-265 \mathrm{~nm}$, in close agreement with that obtained by DLS.

\subsection{In vitro release studies}

The in vitro release studies were conducted for LNS-loaded NPs and the pure drug at $\mathrm{pH} 6.8$ in order to mimic the release pattern in the intestinal environment, as LNS is

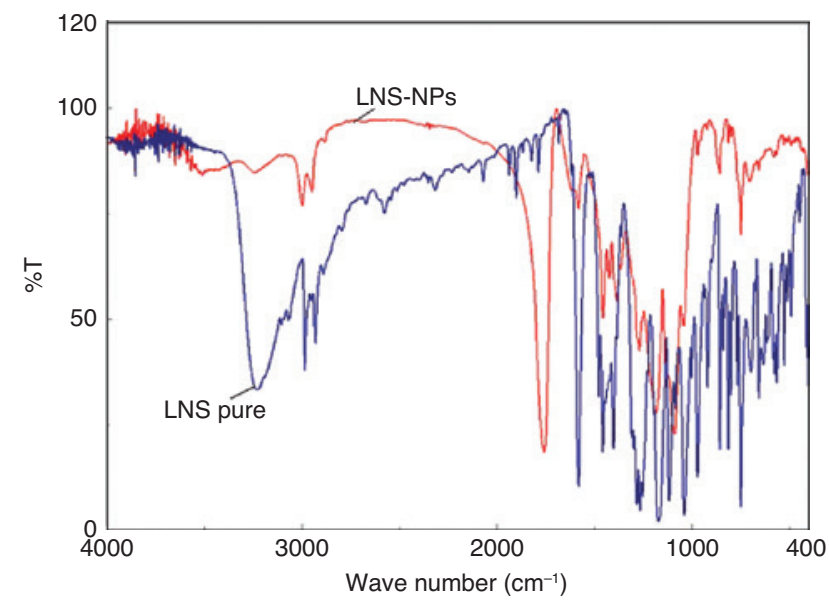

Figure 4: FT-IR spectra of LNS and LNS-NPs. 


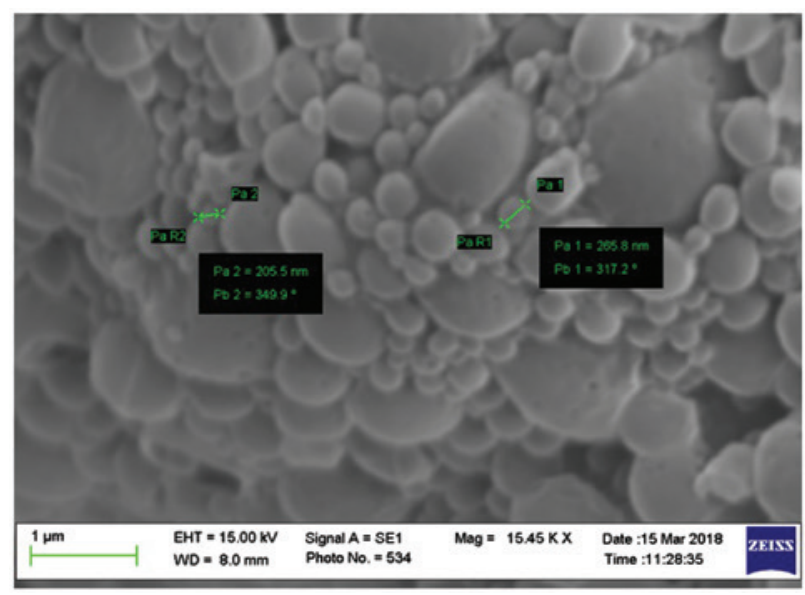

Figure 5: SEM images of LNS-loaded PLGA nanoparticles.

very unstable at lower $\mathrm{pH}$ values. According to the release profile, pure LNS and LNS-loaded NPs were found to be $55.4 \%$ and $90.9 \%$ dissolved after $48 \mathrm{~h}$ of the study, respectively (Figure 6). However, a significant increase in dissolution was observed in LNS-loaded NPs in comparison to the pure drug, which may be attributed to the encapsulation of LNS inside the PLGA matrix. For a drug whose solubility is $\mathrm{pH}$-dependent, the dissolution increases when a $\mathrm{pH}$ modifier is added to the formulation. The addition of $\mathrm{MgO}$ to the formulation leads to an improvement of the solubility [13, 27]. The data obtained from the drug release from the nanoparticles was fitted to different kinetic models to know the release mechanism of LNS from the PLGA matrix. The release mechanism of the drug from LNS-loaded nanoparticles could be consistently fitted to the Higuchi model with a value of $R^{2} \geq 0.9800$.

\subsection{Photodegradation studies}

The percentages of LNS remaining after exposure of pure LNS and LNS-loaded PLGA nanoparticles to $254 \mathrm{~nm}$ UV

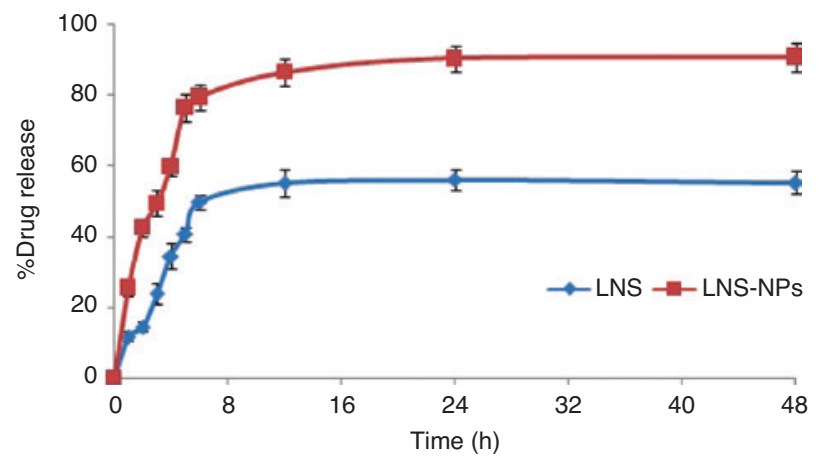

Figure 6: In vitro release profile of LNS-NPs in comparison with that of pure LNS.

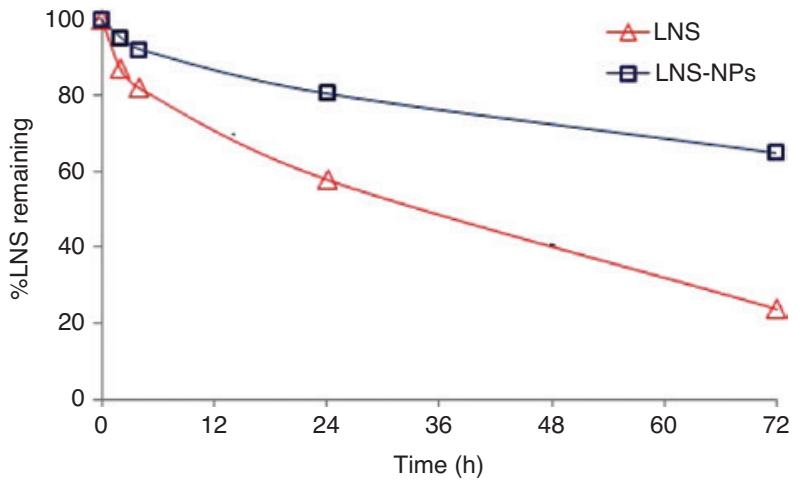

Figure 7: Photodegradation of LNS-NPs in comparison with that of pure LNS after exposure to UV light at $254 \mathrm{~nm}$.

light are shown in Figure 7. The data reveal that the LNS content was reduced from $99.9 \%$ to $24 \%$ in the case of pure LNS powder and from $99.9 \%$ to $65 \%$ in the case of LNS-loaded PLGA nanoparticles after $72 \mathrm{~h}$ of exposure of UV light of $254 \mathrm{~nm}$. This might be due to the light sensitivity of LNS when exposed to short-wavelength UV light $(254 \mathrm{~nm})$. The LNS-loaded PLGA nanoparticles were significantly more stable as compared to pure LNS. To confirm this, LNS-loaded PLGA nanoparticles were exposed to long-wavelength (366 nm) UV light for 1 month, which showed no color change as compared to the pure LNS (Figure 8). The photostability of LNS-loaded PLGA nanoparticles may be attributed to the encapsulation of LNS inside the PLGA polymeric matrix, which protects the drug from light. Moreover, the photostability of LNS-loaded PLGA nanoparticles is enhanced by the presence of the alkalizer $(\mathrm{MgO})$, which provides a $\mathrm{pH}$ of 8.10. Also, the hydrogen bond formed between LNS and the carrier plays a role in the photostabilization of the drug, as hydrogen bonding positively affects the physical and chemical stability [28-30].

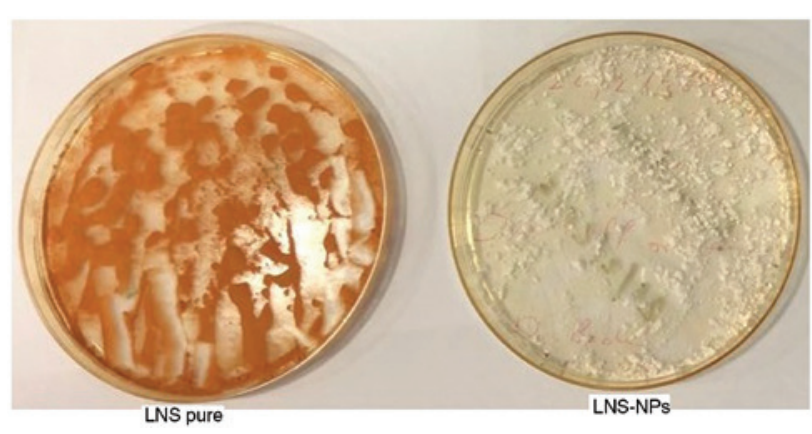

Figure 8: Samples of pure LNS and LNS-NPS after exposure to UV light at $254 \mathrm{~nm}$. 


\subsection{Photodegradation studies by NMR spectroscopy and LC-MS}

A change in the color of pure LNS could be seen in comparison to LNS-loaded PLGA nanoparticles after exposure to UV light at $254 \mathrm{~nm}$ for $72 \mathrm{~h}$ (Figure 9), which clearly demonstrates a stable LNS formulation developed using PLGA. Exposure of pure LNS and LNS-loaded PLGA nanoparticles to UV light at $254 \mathrm{~nm}$ for $72 \mathrm{~h}$ resulted in an almost single degradation product (B) (Figure 10). After purification using column chromatography using silica gel and chloroform/methanol as mobile phase to get rid of the formulation components, the pure compounds were subjected to NMR study using DMSO as solvent. Although the degradation product showed same number of protons and carbons in both ${ }^{1} \mathrm{H}$ and ${ }^{13} \mathrm{C}$ NMR, the pattern of protons showed significant differences (Figure 10). The main differences were observed in the splitting pattern of protons at positions 1-4. These differences indicated that the environment around the protons was changed. LC-MS of the pure LNS and LNS-loaded PLGA nanoparticles exposed to UV light at $254 \mathrm{~nm}$ for $72 \mathrm{~h}$ showed the same $(\mathrm{M}+1)$ peak at $370 \mathrm{~m} / \mathrm{e}$, and the base peak at $369 \mathrm{~m} / \mathrm{e}$ accounted for the unchanged structure of LNS (A) (Figure 10).

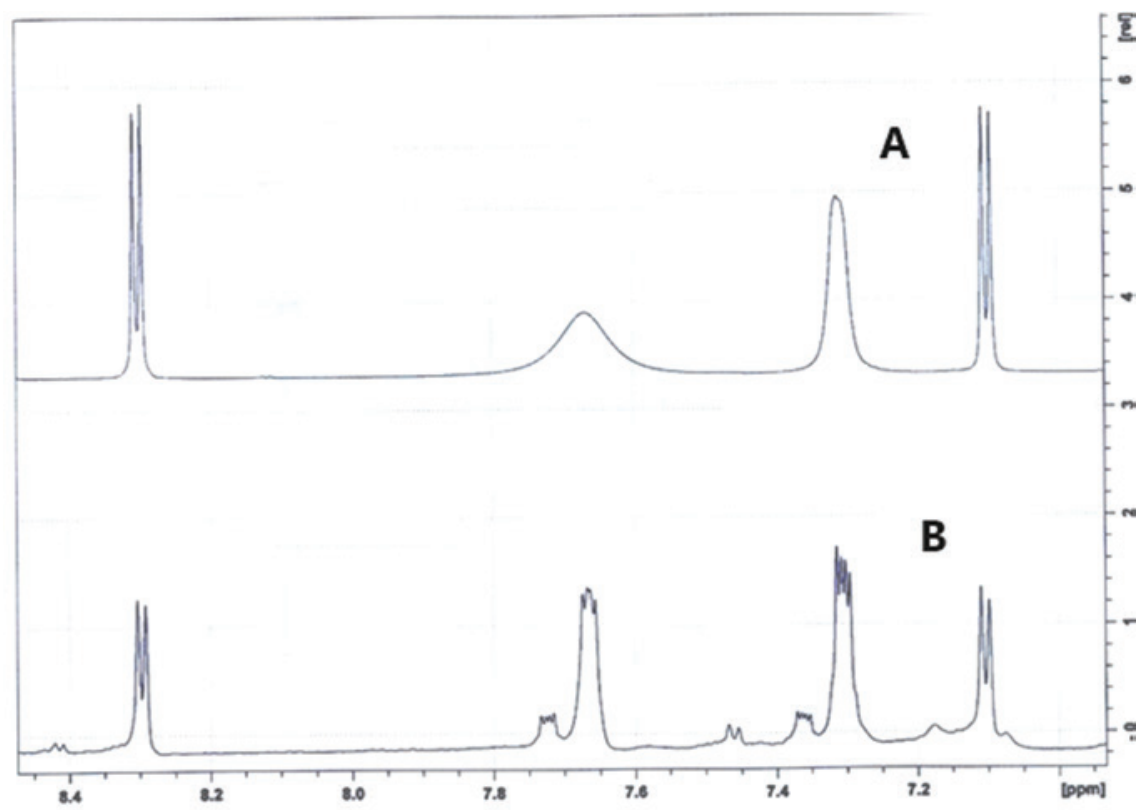

Figure 9: ${ }^{1} \mathrm{H}-\mathrm{NMR}$ splitting pattern of LNS obtained from LNS-NPS exposed to UV light at $254 \mathrm{~nm}(\mathrm{~A})$ and the degradation product of pure LNS exposed to UV light at $254 \mathrm{~nm}$ (B).

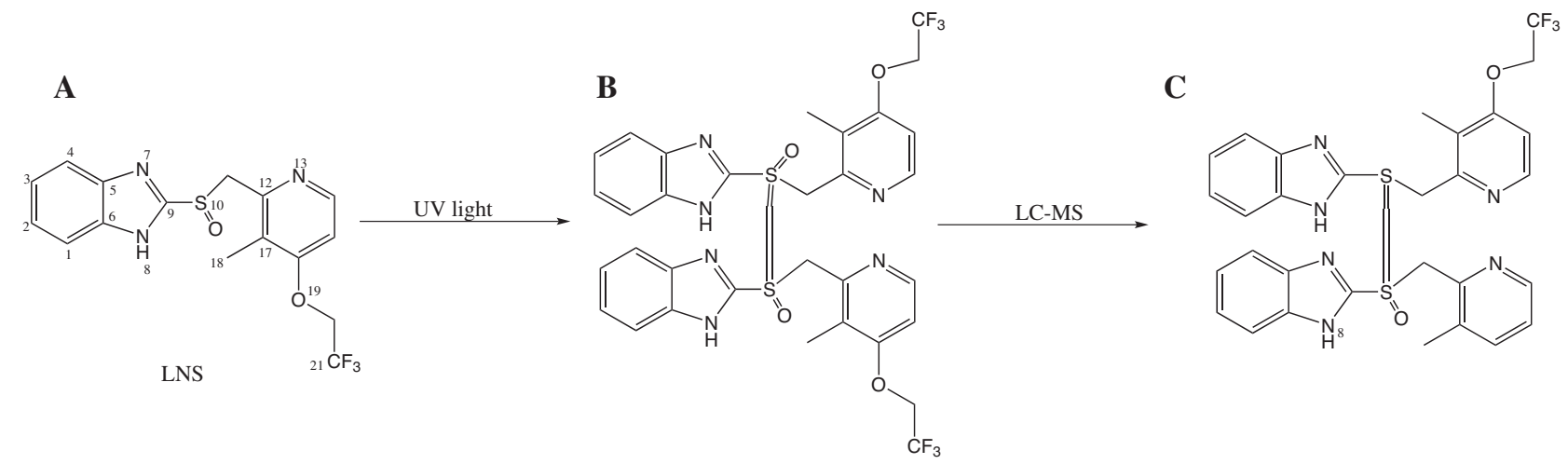

Figure 10: Degradation of LNS by UV radiation and the base peak of the degradation product in LC-MS. (A) LNS; (B) degradation product of non formulated LNS; (C) the base peak of LNS degradation product in LC-MS. 
The base peak of the compound obtained after purification of the degradation product of pure LNS, under the UV light, was observed at $663 \mathrm{~m} / \mathrm{e}$, indicating dimerization of the molecule via oxidation of the sulfur atoms to a higher oxidation level. The base peak (C) consequently resulted from the loss of one oxygen atom and the $\mathrm{OCH}_{2} \mathrm{CF}_{3}$ moiety from the formed dimer (B) (Figure 10).

\section{Conclusion}

In conclusion, LNS-loaded PLGA nanoparticles in the presence of $\mathrm{MgO}$ as alkalizer were successfully developed using the nanoprecipitation/solvent evaporation technique. However, encapsulation of LNS within the PLGA polymeric matrix with the assistance of $\mathrm{MgO}$ resulted in a stable and sustained-release formulation. The resistance to photodegradation was examined by exposing the pure LNS and LNS-loaded PLGA nanoparticles to $254 \mathrm{~nm} \mathrm{UV}$ radiation for $72 \mathrm{~h}$. The intact drug was quantified by HPLC and the degradation products were identified by ${ }^{1} \mathrm{H}$ NMR and LC-MS. The obtained results indicated that the LNSloaded PLGA nanoparticles in the presence of the alkalizer offer a promising formulation to improve the release and the photostability of this sensitive drug.

Funding: This project was supported by the Deanship of Scientific Research at Prince Sattam Bin Abdulaziz University under research project no. 2016/03/6686.

Conflict of interest statement: The authors report no conflict of interest associated with this study.

\section{References}

[1] Battu S, Pottabathini V. Am. J. Anal. Chem. 2015, 6, 145-155.

[2] Barradell LB, Faulds D, McTavish D. Drugs 1992, 44, 225-250.

[3] Wua C, Suna L, Suna J, Yang Y, Renb C, Ai X, Lianb H, He Z. Int. J. Pharm. 2013, 453, 300-306.

[4] Horn JR, Howden CW. Aliment Pharmacol. Ther. 2005, 22, 20-24.

[5] Chono S, Matsui M, Nakamura K, Kasai R. J. Pharm. (Cairo) 2016, 2016, 6131608.
[6] Schmarda A, Dinkhauser P, Gschwentner M, et al. Br. J. Pharmacol. 2000, 129, 598-604.

[7] Zhang X, Sun N, Wu B, Lu Y, Guan T, Wu W. Powder Technol. 2008, 182, 480-485.

[8] Lu Y, Guo T, Qi J, Zhang J, Wu W. AAPS PharmSciTech. 2012, 13, 1222-1229.

[9] Axon AT. J. Clin. Gastroenterol. 1995, 20, S43-S47.

[10] DiGiacinto JL, Olsen KM, Bergman KL, Hoie EB. Ann. Pharmacother. 2000, 34, 600-605.

[11] Roche VF. Am. J. Pharm. Educ. 2006, 70, 101.

[12] Rajab A, Touma M, Rudler H, Afonso C, Seuleiman M. Pharmazie 2013, 68, 749-753.

[13] Alsulays BB, Kulkarni V, Alsheri SM, et al. Drug Dev. Ind. Pharm. 2017, 43, 789-796.

[14] Fang Y, Wang G, Zhang R, Liu Z, Wu X, Cao D. AAPS PharmSciTech. 2014, 15, 513-521.

[15] Lin WJ, Duh YS. Eur. J. Pharm. Biopharm. 2016, 108, 297-303.

[16] Vora C, Patadia R, Mittal K, Mashru R. Sci. Pharm. 2016, 84, 393-408.

[17] Anwer MK, Al-Mansoor MA, Jamil S, Al-Shdefat R, Ansari MN, Shakeel F. Int. J. Biol. Macromol. 2016, 92, 213-219.

[18] Anwer MK, Jamil S, Ansari MJ, Imam F, Shakeel F. Mat. Res. Innov. 2016, 20, 193-197.

[19] Anwer MK, Al-Shdefat R, Ezzeldin E, Alshahrani SM, Alshetaili AS, Iqbal M. Front Pharmacol. 2017, 8, 844.

[20] Park JB, Park Y], Kang CY, Lee BJ. Arch. Pharm. Res. 2015, 38, 839-848.

[21] British pharmacopoeia chemical reference substance information leaflet. Lansoprazole Impurity Standard. Catalogue Number 873. Current Batch: 3188. United Kingdom.

[22] Anwer MK, Mohammad M, Ezzeldin E, Fatima F, Alalaiwe A, Iqbal M. Int. J. Nanomed. 2019, 14, 1587-1595.

[23] Monographs. Medicinal and Pharmaceutical Substances. Lansoprazole Delayed Release Capsule. The United States Pharmacopoeia (USP 29)/ National Formulary (NF 24) Rockville, USA: The United States Pharmacopoeial Convention, 2006.

[24] Aman W, Thoma K. Pharmazie 2003, 58, 877-880.

[25] Loele G, Luca MD, Garofalo A, Ragno G. Drug Deliv. 2017, 24, 33-44.

[26] Suri S, Mirza M, Anwer MK, Alshetaili AS, Alshahrani SM, Ahmed FJ, Iqbal Z. J. Polym. Eng. 2019, 39, 271-278.

[27] Yu M, Sun L, Li E, et al. J. Mol. Struct. 2011, 1005, 70-77.

[28] Tabatar T, Makino T, Kashihara T, Hirai S, Kitamori N, Toguchi H. Drug Dev. Ind. Pharm. 1992, 18, 1437-1447.

[29] Papageorgiou G, Papadimitriou S, Karavas E, Georgarakis E, Docosils A, Bikiaris D. Curr. Drug Deliv. 2009, 6, 101-112.

[30] Guo Z, Lu M, Li Y, Pang H, Lin L, Wu C. J. Pharm. Pharmacol. 2014, 66, 285-296. 